\title{
Blutzuckersenkung bringt wenig - Mythos oder Fakt?
}

\author{
Dies ist klar: Typ-2-Diabetes ist mit Folgeschäden assoziiert. Doch lassen sich diese auch durch eine \\ strenge Glukoseeinstellung meist nicht ganz verhindern. Daher stellen sich viele Fragen wie: Was \\ bringt strenge Einstellung wirklich? Und: Brauchen wir neue Ansätze gegen die Folgeerkrankungen?
}

„Ein hoher Blutzucker ist immer dann als schädlich anzusehen, wenn die Blutzuckersenkung alle diabetischen Folgeschäden verhindern kann", sagte Prof. Peter Nawroth von der Uniklinik in Heidelberg. Doch dies sei nicht wissenschaftlich bewiesen. Die Daten der Diabetes Control and Complications Research Group zeigten sowohl bei Primär- als auch Sekundärprävention der Albuminurie nur einen sehr geringen Effekt einer strengen BZ-Einstellung [1]. Doch die Normalisierung der Nierenfunktion sei für das Überleben doch wohl wichtiger als eine strenge Glukosekontrolle. Bei der Retinopathie habe man errechnen können, dass der $\mathrm{HbA}_{1 \mathrm{c}}$-Wert und die Diabetesdauer nur zu 11\% an der Entstehung beteiligt seien [2]. Und auch in der UKPD-Studie sei die Effizienz bzgl. des Verhinderns von Spätschäden sehr gering. „Wenn aber der Anteil der Hyperglykämien und der Diabetesdauer an der Entstehung der Spätschäden so gering ist, dann sollte man neu nachdenken, was Diabetes ist", so Nawroth.

\section{$\mathrm{HbA}_{1 c}$ nicht über $8,5 \%$}

Diese Daten sprächen dafür, so Nawroth, dass beim Typ-2-Diabetiker bei der Manifestation der Spätschäden noch andere pathogenetische Mechanismen als erhöhte Blutzucker(BZ)werte beteiligt sein müssen. Ergebnisse z.B. der ACCORD-Studie zeigten dann auch, dass eine zu strenge Stoffwechselkontrolle mehr schadet als nutzt. Ähnlich sind die Ergebnisse von Registerstudien [3]. „Doch die Studien lassen auch keinen Zweifel daran, dass ein $\mathrm{HbA}_{1 \mathrm{c}}$-Wert über 8,5\% mit einem erhöhten Risiko für Folgeschäden verbunden ist", so Nawroth. Daher gebe es kein Argument für eine schlechte, aber auch keines für eine normnahe BZ-Einstellung. Die einzige Komplikation, die zu 100\% durch eine BZ-Senkung verhindert werden könne, sei das diabetische Koma. Ansonsten dürfe nur ein partieller Effekt zu erwarten sein. „Somit sei ein hoher Blutzucker nicht immer

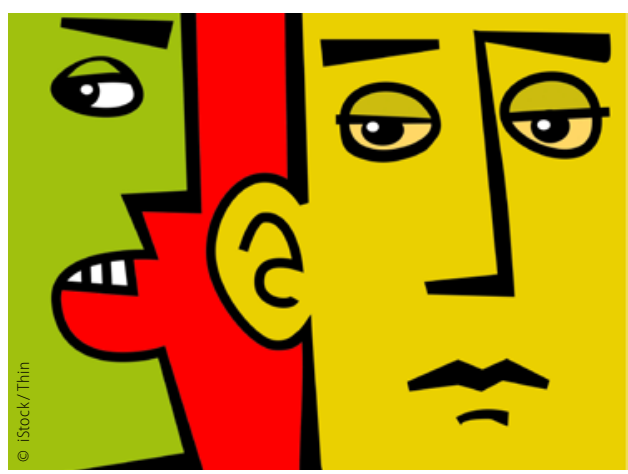
schädlich." Und umgekehrt bedeute ein optimaler $\mathrm{HbA}_{1 \mathrm{c}}$-Wert noch lange keine Normalisierung des Risikos für Folgeschäden. Die Früherkennung eines Typ-2Diabetes ist nach Meinung von Nawroth nicht sinnvoll, da das metabolische Syndrom zwar das Risiko für Typ-2-Diabetes vorhersage. Aber trotz der starken Assoziation mit dem Diabetes sei es kein zuverlässiger Prädiktor für kardiovaskuläre Ereignisse. In der ADDITION-Cambridge-Studie fand sich kein Unterschied bzgl. Mortalität zwischen gescreenten und nicht gescreenten Patienten. Bisher gebe es auch keine ausreichende Evidenz, dass mit einer guten BZ-Einstellung makrovaskuläre Komplikationen verhindert werden bzw. die Prognose quo ad vitam verbessert werden könne [4].

\section{Diabetes ist mehr als Blutzucker}

„Das Fach Diabetologie hat durch die allzu starke Fokussierung auf einen im Blut messbaren Parameter, nämlich die Glukose, Schaden genommen“, so Nawroth. Die Diabetologen hätten sich zur MTA des Laborarztes degradieren lassen. Der Diabetes sei aber mehr als Glukose. Die Erkrankung „Diabetes“ könne man nicht durch Blutanalysen sondern nur durch das Erkennen der intrazellulären Veränderungen verstehen. Das ausschließliche Konzentrieren auf einen Surrogatparameter sei eine Fehlentwicklung. Die eigentliche Erkrankung, die den Folgeschäden zugrunde liege, finde in den Zellen und nicht im Blut statt. Vieles spreche dafür, dass die diabetischen Spätschäden durch eine gestörte enzymatische Detoxifikation reaktiver Metabolite entstehen. Doch die Diabetologie konzentriere sich auf die Entwicklung neuer BZ-Senker und das Interesse an Substanzen, die die Folgeschäden verhindern, sei sehr gering. Der Verlust an wissenschaftlicher Phantasie vernichte sogar Forschungsleistung, so Nawroth. Die den Spätschäden zugrunde liegenden pathogenetischen Mechanismen seien individuell und von Organ zu Organ unterschiedlich, sodass nicht mit einer einzigen Substanz alle diese Schäden verhindert werden können. Zunächst müssten die beteiligten Stoffwechselwege genau analysiert werden, bevor spezifische Wirkstoffe entwickelt werden können. Vorstellbar seien auch neue Substanzen, die schützende Stoffwechselwege stimulieren.

Dr. Peter Stiefelhagen

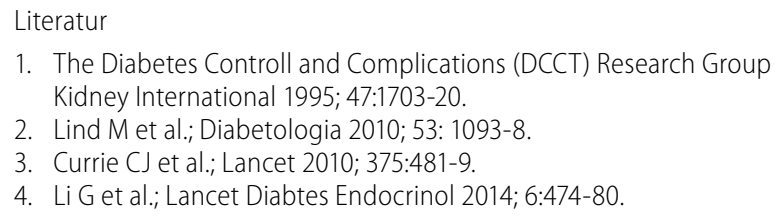

Quelle: Symposium "Mythen in der Diabetologie“, 50. Deutscher Diabeteskongress, 15.5.2015 in Berlin 